\title{
MIRROR SYMMETRY AND RATIONAL CURVES ON QUINTIC THREEFOLDS: A GUIDE FOR MATHEMATICIANS
}

\author{
DAVID R. MORRISON
}

\section{INTRODUCTION}

There has been much recent excitement among mathematicians about a calculation made by a group of string theorists (P. Candelas, X. C. de la Ossa, P. S. Green, and L. Parkes [6]) which purports to give a count of the number of rational curves of fixed degree on a general quintic threefold. The calculation mixes arguments from string theory with arguments from mathematics, and is generally quite difficult to follow for mathematicians.

I believe that I now understand the essential mathematical content of that calculation. It is my purpose in this note to explain my understanding in terms familiar to algebraic geometers. What Candelas et al. actually calculate is a $q$ expansion of a certain function determined by the variation of Hodge structure of some other family of threefolds with trivial canonical bundle. The "mirror symmetry principle" is then invoked to predict that the Fourier coefficients in that expansion should be related to the number of rational curves on a quintic threefold.

One mathematical surprise in this story is a new $q$-expansion principle for functions on the moduli space of Calabi-Yau manifolds. Near points on the boundary of moduli where the monodromy is "maximally unipotent," there turn out to be natural coordinates in which to make $q$-expansions of functions. In this paper, we will discuss these $q$-expansions only in the case of one-dimensional moduli spaces; the general case will be treated elsewhere.

By focusing on this $q$-expansion principle, we place the computation of [6] in a mathematically natural framework. Although there remain certain dependencies on a choice of coordinates, the coordinates used for calculation are canonically determined by the monodromy of the periods, which is itself intrinsic. On the other hand, we have removed some of the physical arguments which were used in the original paper to help choose the coordinates appropriately. The result may be that our presentation is less convincing to physicists.

Received by the editors October 29, 1991.

1991 Mathematics Subject Classification. Primary 14D07; Secondary 14N10, 32G81, 81T40.

This work was partially supported by NSF grant DMS-9103827. 
The plan of the paper is as follows. In $\S 1$ we review variations of Hodge structure, and explain how to define "Yukawa couplings" in Hodge-theoretic terms. (A discussion of Yukawa couplings along the same lines has also been given by Cecotti $[9,10]$.) In $\S 2$ we study the asymptotic behavior of the periods near points with maximally unipotent monodromy. This is applied to find $q$ expansions of the Yukawa couplings in $\S 3$. In $\S 4$ we attempt to describe mirror symmetry in geometric terms. In $\S 5$ we turn to the main example (the family of "quintic-mirrors"), and in $\S 6$ we explain how the mirror symmetry principle predicts from the earlier calculations what the numbers of rational curves on quintic threefolds should be. Several technical portions of the paper have been banished to appendices.

We work throughout with algebraic varieties over the complex numbers, which we often identify with complex manifolds (or complex analytic spaces). If $X$ is a compact complex manifold and $p, q \geq 0$, we define

$$
H^{p, q}(X)=H^{q}\left(\Omega_{X}^{p}\right)=H^{q}\left(\Lambda^{p} \Omega_{X}\right)
$$

where $\Omega_{X}$ is the holomorphic cotangent bundle of $X$. (This is slightly nonstandard.) We extend this definition to the case $p<0, q \geq 0$ by

$$
H^{p, q}(X)=H^{q}\left(\Lambda^{-p} \Theta_{X}\right),
$$

where $\Theta_{X}$ is the holomorphic tangent bundle of $X$. (This is very nonstandard.) The dimension of $H^{p, q}(X)$ is denoted by $h^{p, q}(X)$, or simply by $h^{p, q}$.

\section{VARIATIONS OF HODGE STRUCTURE ARISING FROM FAMILIES OF CALABI-YAU MANIFOLDS}

Recall that a Calabi-Yau manifold is a compact Kähler manifold $X$ of complex dimension $n$ which has trivial canonical bundle, such that the Hodge numbers $h^{k, 0}$ vanish for $0<k<n$. Thanks to a celebrated theorem of Yau [41], every such manifold admits Ricci-flat Kähler metrics.

Any nonzero section of the canonical bundle determines isomorphisms

$$
H^{i}\left(\Theta_{X}\right) \cong H^{i}\left(\Omega_{X}^{n-1}\right) \text {. }
$$

Thus, if $n>1$, then $X$ has no holomorphic vector fields. Moreover, the tangent space to moduli $H^{1}\left(\Theta_{X}\right)$ has dimension $h^{-1,1}=h^{n-1,1}$ and the natural obstruction space for the moduli problem $H^{2}\left(\Theta_{X}\right)$ has dimension $h^{n-1,2}$, which is generally nonzero for $n>2$. However, the theorem of Bogomolov [4], Tian [39], and Todorov [40] says that the moduli problem is in fact unobstructed, and the moduli space is therefore smooth of dimension $h^{n-1,1}$.

We review some facts about Hodge structures and their variation. Good general references for this are Griffiths et al. [21], and Schmid [35]. The $n$th cohomology group of $X$ has a Hodge decomposition

$$
H^{n}(X, \mathbb{C}) \cong \bigoplus_{\substack{p+q=n \\ p, q \geq 0}} H^{p, q}(X) .
$$


(With our conventions, this follows from the Hodge theorem in de Rham cohomology $H_{\mathrm{DR}}^{n}(X)=\bigoplus H_{\bar{\partial}}^{p, q}(X)$ together with the Dolbeault isomorphism $H_{\tilde{\partial}}^{p, q}(X) \cong H^{q}\left(\Omega_{X}^{p}\right)=H^{p, q}(X)$.) The Hodge decomposition can also be described by means of the Hodge filtration

$$
F^{p}(X):=\bigoplus_{p \leq p^{\prime} \leq n} H^{p^{\prime}, n-p^{\prime}}(X) ;
$$

we then have $H^{p, n-p}(X) \cong F^{p}(X) / F^{p+1}(X)$.

The cup product on cohomology composed with evaluation on the canonical orientation class of $X$ determines a bilinear map

$$
\langle\mid\rangle: H^{n}(X, \mathbb{Z}) \times H^{n}(X, \mathbb{Z}) \rightarrow H^{2 n}(X, \mathbb{Z}) \stackrel{\cong}{\rightrightarrows},
$$

called a polarization. There is an associated adjoint map

$$
\operatorname{ad}_{\langle\mid\rangle}: H^{n}(X, \mathbb{Z}) \rightarrow \operatorname{Hom}\left(H^{n}(X, \mathbb{Z}), \mathbb{Z}\right)
$$

defined by $\operatorname{ad}_{\langle\mid\rangle}(x)(y)=\langle x \mid y\rangle$. After tensoring with $\mathbb{C}$ and invoking the Hodge decomposition, the adjoint map induces isomorphisms

$$
\operatorname{ad}_{\langle\mid\rangle}: H^{p, n-p}(X) \cong\left(H^{n-p, p}(X)\right)^{*} .
$$

We now recall a construction which first arose in the study of infinitesimal variations of Hodge structure by Carlson, M. Green, Griffiths, and Harris [8]. The cup product determines a natural map

$$
H^{1}\left(\Theta_{X}\right) \rightarrow \bigoplus \operatorname{Hom}\left(H^{p, q}(X), H^{p-1, q+1}(X)\right)
$$

called the differential of the period map. Iterates of this map are symmetric in their variables; the $n$th iterate of the differential is the induced map

$$
\operatorname{Sym}^{n} H^{1}\left(\Theta_{X}\right) \rightarrow \operatorname{Hom}\left(H^{n, 0}(X), H^{0, n}(X)\right) .
$$

Using the canonical isomorphism $\operatorname{Hom}\left(H^{n, 0}(X), H^{0, n}(X)\right)=\left(H^{n, 0}(X)\right)^{*} \otimes$ $H^{0, n}(X)$ and the isomorphism $H^{0, n}(X) \cong\left(H^{n, 0}(X)\right)^{*}$ induced by the adjoint, we get a map

$$
\operatorname{Sym}^{n} H^{1}\left(\Theta_{X}\right) \rightarrow\left(H^{n, 0}(X)^{*}\right)^{\otimes 2} \text {. }
$$

We call this the unnormalized Yukawa coupling ${ }^{1}$ of $X$.

If we choose an element of $H^{n, 0}(X)^{\otimes 2}$ and evaluate the map (2) on that element, we get a map $\operatorname{Sym}^{n} H^{1}\left(\Theta_{X}\right) \rightarrow \mathbb{C}$ called a normalized Yukawa coupling. The "normalization" is the choice of element of $H^{n, 0}(X)^{\otimes 2}$.

We now analyze these constructions for a family of manifolds. Suppose we are given a quasi-projective variety $C$ and a smooth map $\pi: \mathscr{X} \rightarrow C$ whose

\footnotetext{
${ }^{1}$ This particular Yukawa coupling is probably only interesting in physics if $n=3$. In dimension $n$, what is being computed here is the " $n$-point Yukawa coupling."
} 
fibers are Calabi-Yau manifolds. Suppose also that this family can be completed to a family of varieties $\bar{\pi}: \overline{\mathscr{X}} \rightarrow \bar{C}$, where $\bar{C}$ is a projective compactification of $C$. The fibers of $\bar{\pi}$ may degenerate over the boundary $B:=\bar{C}-C$. We assume that $B$ is a divisor with normal crossings on $\bar{C}$.

For any point $P \in C$ we denote the fiber $\pi^{-1}(P)$ of $\pi$ by $X_{P}$. The KodairaSpencer map $\rho: \Theta_{C, P} \rightarrow H^{1}\left(\Theta_{X_{P}}\right)$ maps the tangent space to $C$ at $P$ to the tangent space to moduli at the point $\left[X_{P}\right]$. If $C$ is actually a moduli space for the fibers of $\mathscr{X}$, the map $\rho$ will be an isomorphism.

The cohomology of the fibers of the map $\pi$ with coefficients in $\mathbb{Z}$ and $\mathbb{C}$ fit together into local systems $R^{n} \pi_{*} \mathbb{Z}$ and $R^{n} \pi_{*} \mathbb{C}$ on $C$. The Hodge filtration becomes a filtration of the vector bundle $\mathscr{F}^{0}:=R^{n} \pi_{*} \mathbb{C} \otimes \mathscr{O}_{C}$ by holomorphic subbundles:

$$
R^{n} \pi_{*} \mathbb{C} \otimes \mathscr{O}_{C}=\mathscr{F}^{0} \supset \mathscr{F}^{1} \supset \cdots \supset \mathscr{F}^{n-1} \supset \mathscr{F}^{n} \supset(0) .
$$

The vector bundle $\mathscr{F}^{0}$ has a natural flat connection $\nabla: \mathscr{F}^{0} \rightarrow \mathscr{F}^{0} \otimes \Omega_{C}$ called the Gauß-Manin connection, whose horizontal sections determine the local system $R^{n} \pi_{*} \mathbb{C}$. The Griffiths transversality property says that $\nabla\left(\mathscr{F}^{p}\right) \subset$ $\mathscr{F}^{p-1} \otimes \Omega_{C}$.

There is a natural extension of this setup over the boundary $B$, which involves the sheaf $\Omega_{\bar{C}}(\log B)$ of logarithmic differentials. (That sheaf is locally generated by $\Omega_{\bar{C}}$ and all elements of the form $d f / f$, where $f=0$ is a local equation of a local component of $B$.) Although the local system $R^{n} \pi_{*} \mathbb{C}$ cannot in general be extended across $B$ in a single-valued way, the Hodge bundles $\mathscr{F}^{p}$ do have natural extensions to bundles $\overline{\mathscr{F}}^{p}$ on $\bar{C}$. And the Gauß-Manin connection $\nabla$ extends to a connection $\bar{\nabla}: \overline{\mathscr{F}}^{0} \rightarrow \overline{\mathscr{F}}^{0} \otimes \Omega_{\bar{C}}(\log B)$ which satisfies $\bar{\nabla}\left(\overline{\mathscr{F}}^{p}\right) \subset \overline{\mathscr{F}}^{p-1} \otimes \Omega_{\bar{C}}(\log B)$. This restriction on the types of poles which $\bar{\nabla}$ may have along $B$ is equivalent to a requirement that the connection $\nabla$ have "regular singular points."

The extended Gauß-Manin connection $\bar{\nabla}$ gives rise to an $\mathscr{Q}_{\bar{C}}$-linear map on the associated gradeds

$$
\tilde{\nabla}: \overline{\mathscr{F}}^{p} / \overline{\mathscr{F}}^{p+1} \rightarrow\left(\overline{\mathscr{F}}^{p-1} / \overline{\mathscr{F}}^{p}\right) \otimes \Omega_{\bar{C}}(\log B) .
$$

To make contact with the $n$th iterate of the differential and the Yukawa coupling, we introduce the sheaf $\Theta_{\bar{C}}(-\log B)$ of vector fields with logarithmic zeros, which is the dual of $\Omega_{\bar{C}}(\log B)$. The map (3) then induces the bundle version of (1)

$$
\Theta_{\bar{C}}(-\log B) \rightarrow \bigoplus \mathscr{H} \operatorname{om}\left(\overline{\mathscr{F}}^{p} / \overline{\mathscr{F}}^{p+1}, \overline{\mathscr{F}}^{p-1} / \overline{\mathscr{F}}^{p}\right) .
$$

When this is iterated $n$ times, it produces a map

$$
\operatorname{Sym}^{n}\left(\Theta_{\bar{C}}(-\log B)\right) \rightarrow \mathscr{H} \operatorname{om}\left(\overline{\mathscr{F}}^{n}, \overline{\mathscr{F}}^{0} / \overline{\mathscr{F}}^{1}\right) \text {. }
$$

The polarizations fit together into a bilinear map of local systems

$$
\langle\mid\rangle: R^{n} \pi_{*} \mathbb{Z} \times R^{n} \pi_{*} \mathbb{Z} \rightarrow R^{2 n} \pi_{*} \mathbb{Z} \cong \mathbb{Z}
$$


whose adjoint map induces an isomorphism $\operatorname{ad}_{\langle\mid\rangle}:\left(\mathscr{F}^{0} / \mathscr{F}^{1}\right) \rightarrow\left(\mathscr{F}^{n}\right)^{*}$. This extends to a map of bundles

$$
\operatorname{ad}_{\langle\mid\rangle}:\left(\overline{\mathscr{F}}^{0} / \overline{\mathscr{F}}^{1}\right) \rightarrow\left(\overline{\mathscr{F}}^{n}\right)^{*}
$$

Using the canonical isomorphism $\mathscr{H}$ om $\left(\overline{\mathscr{F}}^{n}, \overline{\mathscr{F}}^{0} / \overline{\mathscr{F}}^{1}\right)=\left(\overline{\mathscr{F}}^{n}\right)^{*} \otimes\left(\overline{\mathscr{F}}^{0} / \overline{\mathscr{F}}^{1}\right)$ and composing the map (5) with the map (4), we get the Yukawa map

$$
\kappa: \operatorname{Sym}^{n}\left(\Theta_{\bar{C}}(-\log B)\right) \rightarrow\left(\left(\overline{\mathscr{F}}^{n}\right)^{*}\right)^{\otimes 2} \text {. }
$$

If we also specify a section of $\left(\overline{\mathscr{F}}^{n}\right)^{\otimes 2}$, we get a normalized Yukawa map

$$
\kappa^{\text {norm }}: \operatorname{Sym}^{n}\left(\Theta_{\bar{C}}(-\log B)\right) \rightarrow \mathscr{Q}_{\bar{C}} \text {. }
$$

Suppose that $C$ is actually the moduli space for the fibers of $\mathscr{X}$, so that $\rho$ is an isomorphism. If we compose $\rho^{-1}$ with a normalized Yukawa map $\kappa^{\text {norm }}$ we get

$$
\operatorname{Sym}^{n} H^{1}\left(\Theta_{X_{P}}\right) \stackrel{\rho^{-1}}{\longrightarrow} \operatorname{Sym}^{n}\left(\Theta_{C, P}\right) \stackrel{\kappa^{\text {norm }}}{\longrightarrow} \mathscr{O}_{C, P}=\mathbb{C} .
$$

In this way, we exactly recover the corresponding normalized Yukawa coupling.

Candelas et al. [6] typically compute the Yukawa coupling in local coordinates (away from the boundary) as follows. Suppose that $\operatorname{dim} C=1$, and that $\psi$ is a local coordinate defined in an open set $U \subset C$. There is an induced vector field $d / d \psi$, which is a local section of $\Theta_{U}$. Choose a section ${ }^{2} \omega$ of $\mathscr{F}^{n}$ over $U$, and define

$$
\kappa_{\psi \ldots \psi}=\kappa\left(\frac{d}{d \psi}, \ldots, \frac{d}{d \psi}\right) \cdot \omega^{2} .
$$

(The number of $\psi$ 's in the subscript is $n$.) This is a holomorphic function on $U$. If we alter $\omega$ by the gauge transformation $\omega \mapsto f \omega$, then the Yukawa coupling transforms as $\kappa_{\psi \ldots \psi} \mapsto f^{2} \kappa_{\psi \ldots \psi}$. "Normalizing the Yukawa map" is the same thing as "fixing the gauge."

Our primary goal will be to compute the asymptotic behavior of the Yukawa map $\kappa$ in a neighborhood of the boundary $B$.

\section{THE ASYMPTOTIC BEHAVIOR OF THE PERIODS}

For simplicity of exposition, we now specialize to the case in which $C$ is a curve. Let $P \in B$ be a boundary point, and let $T_{P}$ be the monodromy of the local system $R^{n} \pi_{*} \mathbb{Z}$ around $P$. We regard $T_{P}$ as an element of Aut $H^{n}\left(X_{P^{\prime}}, \mathbb{Z}\right)$, where $P^{\prime}$ is a point near $P ; T_{P}$ is determined by analytic continuation along a path which goes once around $P$ in the counterclockwise direction. By the monodromy theorem [24], $T_{P}$ is quasi-unipotent, which means that some power $T_{P}^{k}$ is unipotent. Moreover, the index of unipotency is bounded: we have $\left(T_{P}^{k}-I\right)^{n+1}=0$.

\footnotetext{
${ }^{2}$ To avoid confusion with the cotangent bundle, we denote this section by $\omega$ rather than $\Omega$. However, in appendix $\mathrm{C}$ below, we will revert to the notation $\Omega$ used in [6].
} 
We say that $P$ is a point at which the monodromy is maximally unipotent if the monodromy $T_{P}$ is unipotent, and if $\left(T_{P}-I\right)^{n} \neq 0$. (Thus, the index of nilpotency of $T_{P}-I$ is maximal.) Since $T_{P}-I$ is nilpotent, we can define the logarithm of the monodromy $N=\log \left(T_{P}\right) \in$ End $H^{n}\left(X_{P^{\prime}}, \mathbb{Q}\right)$ by a finite power series

$$
\log \left(T_{P}\right)=\left(T_{P}-I\right)-\frac{\left(T_{P}-I\right)^{2}}{2}+\cdots+(-1)^{n+1} \frac{\left(T_{P}-I\right)^{n}}{n} .
$$

(Rational coefficients are needed in cohomology since rational numbers appear in the power series.) $N$ is also a nilpotent matrix, with the same index of nilpotency as $T_{P}-I$.

Lemma 1. Let $\pi: \mathscr{X} \rightarrow C$ be a one-parameter family of varieties with $h^{n, 0}=1$. Let $P \in B=\bar{C}-C$ be a boundary point at which the monodromy on $R^{n} \pi_{*} \mathbb{Z}$ is maximally unipotent and let $N$ be the logarithm of the monodromy. Then the image of $N^{n}$ is a $\mathbb{Q}$-vector space of dimension one, and the image of $N^{n-1}$ is a $\mathbb{Q}$-vector space of dimension two.

We defer the proof of this lemma to Appendix A.

We say that a basis $g_{0}, g_{1}$ of $\left(\operatorname{Im} N^{n-1}\right) \otimes \mathbb{C} \subset H^{n}\left(X_{P^{\prime}}, \mathbb{C}\right)$ is an adapted basis if $g_{0}$ spans $\left(\operatorname{Im} N^{n}\right) \otimes \mathbb{C}$. (We have extended scalars to $\mathbb{C}$ since certain computational procedures lead more naturally to complex coefficients.) If $g_{0}$, $g_{1}$ is an adapted basis for $\left(\operatorname{Im} N^{n-1}\right) \otimes \mathbb{C}$, then by Poincaré duality, there are homology classes $\gamma_{0}, \gamma_{1} \in H_{n}\left(X_{P^{\prime}}, \mathbb{C}\right)$ such that $\left\langle g_{j} \mid \alpha\right\rangle=\int_{\gamma_{j}} \alpha$ for any $\alpha \in$ $H^{n}\left(X_{P^{\prime}}, \mathbb{C}\right)$. Here we denote the evaluation of cohomology classes on homology classes by using an integral sign, since that evaluation is often accomplished by integration.

Proposition. Let $\gamma_{0}, \gamma_{1}$ be the homology classes determined by an adapted basis $g_{0}, g_{1}$ of $\left(\operatorname{Im} N^{n-1}\right) \otimes \mathbb{C}$. Define a constant $m$ by $N g_{1}=m g_{0}$. Let $\bar{U}$ be a small neighborhood of $P$, and let $z$ be a coordinate on $\bar{U}$ centered at $P$. Let $\omega$ be a nonzero section of $\overline{\mathscr{F}}^{n}$ over $\bar{U}$. Then

(1) $\int_{\gamma_{0}} \omega$ extends to a single-valued function on $\bar{U}$.

(2) $\int_{\gamma_{1}} \omega$ is not single-valued. However, we can write

$$
\frac{\frac{1}{m} \int_{\gamma_{1}} \omega}{\int_{\gamma_{0}} \omega}=\frac{\log z}{2 \pi i}+\text { single-valued function. }
$$

Proof. Any $g \in H^{n}\left(X_{P^{\prime}}, \mathbb{C}\right)$ can be extended to a section $g(z)$ of the local system over $U=\bar{U}-P$, which may be multi-valued. But by the nilpotent orbit theorem [35], $\exp \left(-\frac{\log z}{2 \pi i} N\right) g(z)$ extends to a single-valued section.

Since $\omega$ is single-valued,

$$
\left\langle\exp \left(-\frac{\log z}{2 \pi i} N\right) g(z) \mid \omega\right\rangle
$$


will also be single-valued. Now $g_{j} \in\left(\operatorname{Im} N^{n-1}\right) \otimes \mathbb{C}$ implies that $N^{2} g_{j}=0$ for $j=1,2$. The series needed for $\exp$ in this case is thus rather simple:

$$
\begin{aligned}
\exp \left(-\frac{\log z}{2 \pi i} N\right) g_{j}(z) & =\left(I-\frac{\log z}{2 \pi i} N\right) g_{j}(z) \\
& =g_{j}(z)-\frac{\log z}{2 \pi i} N g_{j}(z) .
\end{aligned}
$$

We conclude that

$$
\int_{\gamma_{0}} \omega=\left\langle g_{0}(z) \mid \omega\right\rangle
$$

and

$$
\int_{\gamma_{1}} \omega-m \frac{\log z}{2 \pi i} \int_{\gamma_{0}} \omega=\left\langle g_{1}(z)-\frac{\log z}{2 \pi i} m g_{0}(z) \mid \omega\right\rangle
$$

are single-valued functions. Q.E.D.

Corollary. Let $\gamma_{0}, \gamma_{1}$ be the homology classes determined by an adapted basis $g_{0}, g_{1}$ of $\left(\operatorname{Im} N^{n-1}\right) \otimes \mathbb{C}$, as in the proposition. The function

$$
t:=\frac{\frac{1}{m} \int_{\gamma_{1}} \omega}{\int_{\gamma_{0}} \omega}
$$

gives a natural parameter on the universal cover $\widetilde{U}$ of $U$ called a quasi-canonical parameter, and

$$
q:=e^{2 \pi i t}
$$

gives a natural coordinate on $\bar{U}$ called a quasi-canonical coordinate. These are independent of the choice of $\omega$. We have

$$
\frac{d}{d t}=2 \pi i q \frac{d}{d q}
$$

either of which serves as a local generator of the sheaf $\Theta_{\bar{C}}(-\log B)$.

Moreover, under a change of adapted basis $\left(g_{0}, g_{1}\right) \mapsto\left(a g_{0}, b g_{0}+c g_{1}\right)$, we have

$$
m \mapsto \frac{c}{a} m, \quad t \mapsto t+\frac{b}{m c}, \quad \text { and } \quad q \mapsto e^{2 \pi i b / m c} q .
$$

Therefore, $t$ is uniquely determined up to an additive constant, and $q$ is uniquely determined up to a multiplicative constant.

We can normalize further if we take the integral structure into account. We call $g_{0}, g_{1}$ a good integral basis of $\operatorname{Im} N^{n-1}$ if $g_{0}$ is a generator of $\operatorname{Im} N^{n} \cap$ $H^{n}\left(X_{P^{\prime}}, \mathbb{Z}\right)$, and $g_{1}$ is an indivisible element of $H^{n}\left(X_{P^{\prime}}, \mathbb{Z}\right)$ which can be written as $g_{1}=\frac{1}{\lambda} N^{n-1} g$ for some $\lambda>0$ and some $g \in H^{n}\left(X_{P^{\prime}}, \mathbb{Z}\right)$ such that $\left\langle g_{0} \mid g\right\rangle=1$. Notice that a good integral basis is an adapted basis.

The next lemma, which is based on some work of Friedman and Scattone [17], will be proved in Appendix A. 
Lemma 2. Good integral bases exist. If $g_{0}, g_{1}$ and $g_{0}^{\prime}, g_{1}^{\prime}$ are good integral bases, then

$$
\begin{aligned}
& g_{1}^{\prime}=k g_{0}+(-1)^{\ell} g_{1}, \\
& g_{0}^{\prime}=(-1)^{\ell} g_{0},
\end{aligned}
$$

for some integers $k$ and $\ell$.

Since $(T-I)^{2}=0$ on $\operatorname{Im} N^{n-1}$, we have the simple formula $N=T-I$ on that space. In particular, when restricted to $\operatorname{Im} N^{n-1}$, the map $N$ is defined over the integers. Thus, if $g_{0}, g_{1}$ is a good integral basis and we write $N g_{1}=$ $m g_{0}$, then $m$ is an integer. Note that $m$ is independent of the choice of good integral basis.

Corollary. Let $g_{0}, g_{1}$ be a good integral basis, and define an integer $m$ by $N g_{1}=m g_{0}$. Then the quasi-canonical coordinate $q$ formed from this basis is unique up to multiplication by an $|m|$ th root of unity.

We call $q$ a canonical coordinate and $t$ a canonical parameter under these circumstances. These are actually unique if $|m|=1$; in this case, we say that the monodromy is small.

\section{The $q$-EXPANSION OF THE YUKAWA COUPLING}

The first example of the construction of the previous section is furnished by the classical theory of periods of elliptic curves. Let $\pi: \mathscr{X} \rightarrow U$ be a family of smooth elliptic curves over a punctured disk $U$ which can be completed to a family $\bar{\pi}: \overline{\mathscr{X}} \rightarrow \bar{U}$ with a singular fiber over the boundary point $P=\bar{U}-U$. The point $P$ is called a cusp.

Let $P^{\prime} \in U$, and suppose there is a symplectic basis $\gamma_{0}, \gamma_{1}$ of the first homology group $H_{1}\left(X_{P^{\prime}}, \mathbb{Z}\right)$ such that the monodromy $T_{P}$ acts as

$$
\begin{aligned}
& T_{P}\left(\gamma_{0}\right)=\gamma_{0}, \\
& T_{P}\left(\gamma_{1}\right)=\gamma_{0}+\gamma_{1} .
\end{aligned}
$$

(The basis is symplectic if $\gamma_{0} \cap \gamma_{1}=1$.) This easily implies that $P$ is a maximally unipotent boundary point, that $\gamma_{0}, \gamma_{1}$ is the homology basis dual to a good integral basis, and that $m=1$.

For a fixed holomorphic one-form $\omega$ on $X_{P^{\prime}}$, the numbers $\left(\int_{\gamma_{0}} \omega, \int_{\gamma_{1}} \omega\right)$ were classically known as the periods of the elliptic curve $X_{P^{\prime}}$. By varying the one-form, the periods can be normalized to take the form $(1, \tau)$. The invariant way to formulate this is to define

$$
\tau=\frac{\int_{\gamma_{1}} \omega}{\int_{\gamma_{0}} \omega} .
$$

This function $\tau$ can be regarded as a map from the universal cover $\widetilde{U}$ of $U$ to the upper half-plane $\mathbb{H}$. (The image lies in the upper half-plane since the basis is symplectic.) 
The monodromy transformation $T_{P}$ induces the map

$$
\tau \mapsto \tau+1 .
$$

Thus, functions $f$ defined on $U$ pull back to functions $\tilde{f}$ on $\widetilde{U}$ which are invariant under (6). It follows that any such function has a Fourier series

$$
\tilde{f}(\tau)=\sum_{n \in \mathbb{Z}} a_{n} e^{2 \pi i n \tau} .
$$

If expressed in terms of the natural coordinate $q=e^{2 \pi i \tau}$ on $U$, this is called a q-expansion, and it takes the form

$$
f(q)=\sum_{n \in \mathbb{Z}} a_{n} q^{n} .
$$

If $f$ has a holomorphic extension across the cusp $P$, the only terms appearing in this sum are those with $n \geq 0$.

What we have shown in $\S 2$ is that this classical construction generalizes to functions defined near a maximally unipotent boundary point $P$ of a CalabiYau moduli space (at least when that space has dimension one). Fix a good integral basis, which determines a canonical coordinate $q$ and a canonical parameter $t$. The monodromy transformation $T_{P}$ acts on $t$ by $t \mapsto t+1$. Therefore, any function $f$ defined near $P$ which is holomorphic at $P$ will have a $q$-expansion

$$
f(q)=\sum_{n=0}^{\infty} a_{n} q^{n},
$$

which can also be regarded as a Fourier series

$$
\widetilde{f}(t)=\sum_{n=0}^{\infty} a_{n} e^{2 \pi i n t}
$$

in $t$. These expressions are unique if $|m|=1$, i.e., if the monodromy is small.

In order to obtain a $q$-expansion of the Yukawa coupling, we must normalize that coupling. But there is a natural choice of normalization determined by a good integral basis. To see this, note that any good integral basis $g_{0}, g_{1}$ determines a section $\left(\int_{\gamma_{0}}\right)^{-1} \in H^{0}\left(\bar{U},\left(\overline{\mathscr{F}}^{n}\right)\right)$ by

$$
\left(\int_{\gamma_{0}}\right)^{-1}:=\frac{\omega}{\int_{\gamma_{0}} \omega}
$$

for any nonzero $\omega \in H^{0}\left(\bar{U}, \bar{F}^{n}\right)$. By Lemma 2, a change in good integral basis may change the sign of $\left(\int_{\gamma_{0}}\right)^{-1}$, but the induced section $\left(\int_{\gamma_{0}}\right)^{-2} \in$ $H^{0}\left(\bar{U},\left(\bar{F}^{n}\right)^{\otimes 2}\right)$ is independent of the choice of good integral basis.

We thus have a very natural normalization for the Yukawa map in $\bar{U}$. We also have a natural parameter $t$ with which to compute, such that $d / d t$ is 
a generator of $\Theta_{\bar{U}}(-\log B)$. So we can define the mathematically normalized Yukawa coupling $\kappa_{t \ldots t}$ by the formula

$$
\kappa_{t \ldots t}=\kappa\left(\frac{d}{d t}, \ldots, \frac{d}{d t}\right) \cdot\left(\int_{\gamma_{0}}\right)^{-2} .
$$

This mathematically normalized Yukawa coupling $\kappa_{t \ldots t}$ is an intrinsically defined function on a neighborhood of the boundary. (It is canonically determined by our choice of maximally unipotent boundary point; however, it could conceivably change if the boundary point changes.) The function $\kappa_{t \ldots t}$ therefore has a $q$-expansion

$$
\kappa_{t \ldots t}=a_{0}+a_{1} q+a_{2} q^{2}+\cdots,
$$

which can also be regarded as a Fourier expansion in the parameter $t$ :

$$
\kappa_{t \ldots t}=a_{0}+a_{1} e^{2 \pi i t}+a_{2} e^{4 \pi i t}+\cdots .
$$

These expressions are unique if the monodromy is small.

\section{MIRROR SYMMETRY}

In this section I will attempt to outline the mirror symmetry principle in mathematical terms, and describe some of the mathematical evidence for it. I apologize to physicists for my misrepresentations of their ideas, and I apologize to mathematicians for the vagueness of my explanations.

Gepner [18] has conjectured that there is a one-to-one correspondence between $N=2$ superconformal field theories with $c=3 n$, and Calabi-Yau manifolds $X$ of dimension $n$ equipped with some "extra structure" $S$. (This correspondence can be realized concretely in a number of important cases using work of Greene, Vafa, and Warner [20], Martinec [27, 28], and others.) A precise geometric description of the extra structure $S$ has not yet been given. It appears to involve specifying a class in $U / \Gamma$, where $U \subset H^{1,1}(X)$ is some open set, and $\Gamma$ is some group of automorphisms of $U$. What is clear about this extra structure is how to perturb it: first-order deformations of $S$ correspond to elements of $H^{1,1}(X)$.

An instructive example is the case in which $X$ is an elliptic curve. In that case, as shown in [12] and [1], one takes $U \subset H^{1,1}(X) \cong \mathbb{C}$ to be the upper half-plane, and $\Gamma=\operatorname{SL}(2, \mathbb{Z})$. Thus, the extra structure $S$ represents a point in the $j$-line, or equivalently, a choice of a second elliptic curve.

We specialize now to the case of dimension $n=3$. The space of firstorder deformations of the superconformal field theory can be decomposed as ${ }^{3}$

\footnotetext{
${ }^{3}$ It has become common in the physics literature to use $H^{2,1}(X)$ in place of $H^{1}\left(\Theta_{X}\right)$, largely because of the success of Candelas [5] and others in computing Yukawa couplings on $H^{2,1}$. In order to get the correct answer in families, however, we must return to the original analysis of Strominger and Witten [37] and work with Yukawa couplings on $H^{1}\left(\Theta_{X}\right)$. The point is that while $H^{1}\left(\Theta_{X}\right)$ and $H^{2,1}(X)$ are isomorphic for a Calabi-Yau threefold, they are not canonically isomorphic. This affects the bundles over the moduli space to which they belong.
} 
$H^{1}\left(\Theta_{X}\right) \oplus H^{1}\left(\Omega_{X}\right)$, with $H^{1}\left(\Theta_{X}\right)=H^{-1,1}(X)$ corresponding to first-order deformations of the complex structure on $X$, and $H^{1}\left(\Omega_{X}\right)=H^{1,1}(X)$ corresponding to first-order deformations of the extra structure $S$. These first-order deformations are called marginal operators in the physics literature.

Specifying a superconformal field theory of this type also determines cubic forms $\operatorname{Sym}^{3} H^{-1,1}(X) \rightarrow \mathbb{C}$ and $\operatorname{Sym}^{3} H^{1,1}(X) \rightarrow \mathbb{C}$. The cubic form on $H^{-1,1}$ is the Yukawa coupling described in $\S 1$, normalized in a way specified by the physical theory. From a mathematical point of view, this is determined by the variation of Hodge structure plus the choice of normalization. This cubic form depends on the complex structure of $X$, but should be independent of the "extra structure" $S$.

The cubic form on $H^{1,1}$ lacks a precise geometric description at present. By work of Dine, Seiberg, Wen, and Witten [13] and Distler and Greene [14], it is known to have an expression of the form

$$
\sum_{k=0}^{\infty} \sigma_{k} e^{-k R}
$$

where $R$ is a complex parameter which depends on the extra structure $S$. The real part of $R$ is related to the "radius" in the physical theory in such a way that $\operatorname{Re} R \rightarrow \infty$ is the "large radius limit." The leading coefficient $\sigma_{0}$ is the natural topological product $\operatorname{Sym}^{3} H^{1,1}(X) \rightarrow \mathbb{C}$. (In other words, the cubic form on $H^{1,1}$ approaches the topological product in the large radius limit.) The higher coefficients $\sigma_{k}$ are supposed to be related in some well-defined way to the numbers of rational curves of various degrees on the generic deformation of $X$ (assuming those numbers are finite). One of the important unsolved problems in the theory is to determine this relationship precisely.

As was first noticed by Dixon [15, p. 118], and later developed by Lerche, Vafa, and Warner [25] and others, the identification of one piece of the superconformal field theory with $H^{1,1}(X)$ and the other piece with $H^{-1,1}(X) \cong$ $H^{2,1}(X)$ involves an arbitrary choice, and the theory is also consistent with making the opposite choice. Moreover, as we will describe below, there are examples in which the Gepner correspondence can be realized for both choices. But except in the very rare circumstance that the Hodge numbers $h^{1,1}$ and $h^{2,1}=\operatorname{dim} H^{-1,1}$ coincide, changing the choice necessarily involves changing the Calabi-Yau threefold $X$. The new threefold $X^{\prime}$ will have a completely different topology from the old: in fact, the Hodge diamond is rotated by $90^{\circ}$ when passing from one to the other.

This leads to a mathematical version of the mirror symmetry conjecture: To each pair $(X, S)$ consisting of a Calabi-Yau threefold $X$ together with some extra structure $S$ there should be associated a "mirror pair" $\left(X^{\prime}, S^{\prime}\right)$ which comes equipped with natural isomorphisms $H^{-1,1}(X) \cong H^{1,1}\left(X^{\prime}\right)$ and $H^{1,1}(X) \stackrel{\cong}{\rightrightarrows} H^{-1,1}\left(X^{\prime}\right)$ that are compatible with the cubic forms. 
Even in this rather imprecise ${ }^{4}$ form, the conjecture as stated is easily refuted: There exist rigid Calabi-Yau threefolds, which have $h^{2,1}=0$ (see Schoen [36] for an example). Any mirror of such a threefold would have $h^{1,1}=0$, and so could not be Kähler. A potentially correct version of the conjecture, even less precise, begins: "To most pairs $(X, S)$, including almost all of interest in physics, there should be associated ...."

It is tempting to speculate that the theory should be extended to non-Kähler threefolds as in Reid's fantasy [31], which might rescue the conjecture in its original form. Alternatively, Aspinwall and Lütken [2] suggest that the Gepner correspondence (and hence the mathematical version of mirror symmetry) should only hold in the large radius limit. Since no "limits" can be taken in the rigid case, a mathematical mirror construction would not be expected there.

To be presented with a conjecture which has been only vaguely formulated is unsettling to many mathematicians. Nevertheless, the mirror symmetry phenomenon appears to be quite widespread, so it seems important to make further efforts to find a precise formulation. In fact, there are at least four major pieces of mathematically significant evidence for mirror symmetry.

(i) Greene and Plesser [19] have studied a case in which there are very solid physics arguments which tie the pair $(X, S)$ to the corresponding superconformal field theory (as predicted by Gepner). The Calabi-Yau threefolds in question are desingularizations of quotients of Fermat-type weighted hypersurfaces by certain finite groups (including the trivial group). For each pair $(X, S)$ of this type, Greene and Plesser were able to find the corresponding mirror pair $\left(X^{\prime}, S^{\prime}\right)$ by analyzing the associated superconformal field theories. It turns out that the pairs are related by taking quotients: $X^{\prime}$ is a desingularization of $X / G$ for some symmetry group $G$. By deformation arguments, the mirror symmetry phenomenon persists in neighborhoods of $(X, S)$ and $\left(X^{\prime}, X^{\prime}\right)$. Roan [34] subsequently gave a direct mathematical proof that the predicted isomorphisms between $H^{-1,1}$ and $H^{1,1}$ groups exist in this situation.

(ii) Candelas, Lynker, and Schimmrigk [7] have computed the Hodge numbers for a large class of Calabi-Yau threefolds which are desingularizations of hypersurfaces in weighted projective spaces. They put some extra constraints on the form of the equation, and found about 6000 types of threefolds satisfying their conditions. The set of pairs $\left(h^{1,1}, h^{2,1}\right)$ obtained from these examples is very nearly (but not precisely) symmetric with respect to the interchange $h^{1,1} \leftrightarrow h^{2,1}$. Since there is no a priori reason that the mirror of a desingularized weighted hypersurface should again be a desingularized weighted hypersurface, this is consistent with the conjecture and supports it quite strongly.

\footnotetext{
${ }^{4}$ Among the things not properly defined from a mathematical viewpoint, we must include the normalization of the Yukawa coupling, the complex parameter $R$ (which depends on the "extra structure" $S$ ), and the higher coefficients $\sigma_{k}$.
} 
(iii) Aspinwall, Lütken, and Ross [3] (see also [1]) have carefully studied a particular mirror pair $(X, S),\left(X^{\prime}, S^{\prime}\right)$. They put $X$ in a family $\mathscr{X}=$ $\left\{X_{t}\right\}$ which has a degenerate limit as $t$ approaches 0 . Some heuristics were used in choosing the family $\mathscr{X}$, in an attempt to ensure that the limit as $t \rightarrow 0$ would correspond to the "large radius limit" for the mirror $\left(X^{\prime}, S^{\prime}\right)$. Aspinwall et al. then computed the limiting behavior of the cubic form on $H^{-1,1}\left(X_{t}\right)$, and showed that it coincides with the topological product $\sigma_{0}^{\prime}$ on $H^{1,1}\left(X^{\prime}\right)$, as predicted by the conjecture. (Actually, there is a normalization factor which was not computed, but the agreement is exact up to this normalization.)

(iv) The work of Candelas, de la Ossa, P. Green, and Parkes [6] being described in this paper goes further, and computes the other coefficients in an asymptotic expansion. This will be explained in more detail in the next two sections.

\section{THE QUINTIC-MIRROR FAMILY}

We now describe a certain one-parameter family of Calabi-Yau threefolds constructed by Greene and Plesser [19], as amplified by Candelas et al. [6]. Begin with the family of quintic threefolds $\mathscr{Q}_{\psi}=\left\{\vec{x} \in \mathbb{P}^{4} \mid p_{\psi}(\vec{x})=0\right\}$ defined by the polynomial

$$
p_{\psi}:=\sum_{k=1}^{5} x_{k}^{5}-5 \psi \prod_{k=1}^{5} x_{k}
$$

Let $\boldsymbol{\mu}_{5}$ be the multiplicative group of 5 th roots of unity, and let

$$
\widetilde{G}:=\left\{\vec{\alpha}=\left(\alpha_{1}, \ldots, \alpha_{5}\right) \in\left(\boldsymbol{\mu}_{5}\right)^{5} \mid \prod_{k=1}^{5} \alpha_{k}=1\right\}
$$

act on $\mathbb{P}^{4}$ by $\vec{\alpha}: x_{i} \mapsto \alpha_{i} \cdot x_{i}$. There is a "scalar" subgroup of order 5 which acts trivially; let $G=\widetilde{G} /\{$ scalars $\}$ be the image of $\widetilde{G}$ in $\operatorname{Aut}\left(\mathbb{P}^{4}\right) . G$ is a group which is abstractly isomorphic to $(\mathbb{Z} / 5 \mathbb{Z})^{3}$.

The action of $G$ preserves the threefold $\mathscr{Q}_{\psi}$; let $\eta: \mathscr{Q}_{\psi} \rightarrow \mathscr{Q}_{\psi} / G$ denote the quotient map. For each pair of distinct indices $i, j$, the set of 5 points

$$
S_{i j}:=\left\{x_{i}^{5}+x_{j}^{5}=0, x_{\ell}=0 \text { for all } \ell \neq i, j\right\} \subset \mathscr{Q}_{\psi}
$$

is preserved by $G$, and there is a group $G_{i j} \subset G$ of order 25 which is the stabilizer of each point in the set. The image $S_{i j} / G$ is a single point $p_{i j} \in$ $\mathscr{Q}_{\psi} / G$. In addition, for each triple of distinct indices $i, j, k$, the curve

$$
\widetilde{C}_{i j k}:=\left\{x_{i}^{5}+x_{j}^{5}+x_{k}^{5}=0, x_{\ell}=0 \text { for all } \ell \neq i, j, k\right\} \subset \mathscr{Q}_{\psi}
$$

is preserved by $G$. There is a subgroup $G_{i j k} \subset G$ of order 5 which is the stabilizer of every point in $\widetilde{C}-\eta^{-1}\left(\left\{p_{i j}, p_{j k}, p_{i k}\right\}\right)$. The image $C_{i j k}=\widetilde{C}_{i j k} / G$ 
is a smooth curve in $\mathscr{Q}_{\psi} / G$. The action of $G$ is free away from the curves $\widetilde{C}_{i j k}$.

The quotient space $\mathscr{Q}_{\psi} / G$ has only canonical singularities. At most points of $C_{i j k}$, the surface section of the singularity is a rational double point of type $A_{4}$, but at the points $p_{i j}$ the singularity is more complicated: three of the curves of $A_{4}$-singularities meet at each $p_{i j}$. By a theorem of Markushevich [26, Prop. 4] and Roan [32, Prop. 2], these singularities can be resolved to give a Calabi-Yau manifold $\mathscr{W}_{\psi}$. There are choices to be made in this resolution process; we describe a particular choice in Appendix B. (By another theorem of Roan [33, Lemma 4], any two resolutions differ by a sequence of flops.)

For any $\alpha \in \boldsymbol{\mu}_{5}$, there is a natural isomorphism between $\mathscr{Q}_{\alpha \psi} / G$ and $\mathscr{Q}_{\psi} / G$ induced by the map

$$
\left(x_{1}, x_{2}, x_{3}, x_{4}, x_{5}\right) \mapsto\left(\alpha^{-1} x_{1}, x_{2}, x_{3}, x_{4}, x_{5}\right) .
$$

This extends to an isomorphism between $\mathscr{W}_{\alpha \psi}$ and $\mathscr{W}_{\psi}$, provided that we have resolved singularities in a compatible way. We verify in Appendix B that the choices in the resolution can be made in a sufficiently natural way that this isomorphism is guaranteed to exist.

Thus, $\lambda:=\psi^{5}$ is a more natural parameter to use for our family. We define the quintic-mirror family to be

$$
\left\{\mathscr{W}_{\sqrt[5]{\lambda}}\right\} \rightarrow\{\lambda\} \cong \mathbb{C}
$$

This has a natural compactification to a family over $\mathbb{P}^{1}$, with boundary $B=$ $\mathbb{P}^{1}-\mathbb{C}=\{\infty\}$.

The computation made by Candelas et al. [6] shows that the monodromy at $\infty$ is maximally unipotent, and that $m=1$, i.e., that the monodromy is small. (We explain in Appendix $C$ how this follows from [6].) The key computation in [6] is an explicit calculation of the $q$-expansion of the mathematically normalized Yukawa coupling. Candelas et al. find that the $q$-expansion begins:

$$
\kappa_{t t t}=5+2875 e^{2 \pi i t}+4876875 e^{4 \pi i t}+\cdots .
$$

In fact, they have computed at least 10 coefficients.

\section{MiRRoR MOONSHINE?}

Greene and Plesser [19], using arguments from superconformal field theory, have identified the family of quintic-mirrors $\left\{\mathscr{W}_{\sqrt[5]{\lambda}}\right\}$ as the "mirror" of the family of smooth quintic threefolds $\left\{\mathscr{M}_{z}\right\}$. Note that the Hodge numbers satisfy

$$
\begin{aligned}
& h^{1,1}(\mathscr{M})=1, \quad h^{2,1}(\mathscr{M})=101, \\
& h^{1,1}(\mathscr{W})=101, \quad h^{2,1}(\mathscr{W})=1 .
\end{aligned}
$$

According to the mirror symmetry conjecture, varying the complex structure in the family $\left\{\mathscr{W}_{\sqrt[5]{\lambda}}\right\}$ should correspond to varying the "extra structure" $S$ on a fixed smooth quintic threefold $\mathscr{M}$. These are both one-parameter variations. 
Candelas et al. [6], arguing from physical principles, propose an identification of the Yukawa coupling of the quintic-mirrors with the cubic form on $H^{1,1}(\mathscr{M})$. In terms of the mathematical framework established here, that identification involves four assertions:

(i) The isomorphism $H^{-1,1}(\mathscr{W}) \rightarrow H^{1,1}(\mathscr{M})$ defined by $d / d t \mapsto[H]$ (where $d / d t \in H^{-1,1}(\mathscr{W}$ ) is the vector field defined by the canonical parameter $t$, and $[H] \in H^{1,1}(\mathscr{M})$ is the class of a hyperplane section of $\mathscr{M})$ is the isomorphism which is predicted by the mathematical version of the mirror symmetry conjecture.

(ii) The mathematically normalized Yukawa coupling $\kappa_{t t t}$ on $H^{-1,1}(\mathscr{W})$ is the correctly normalized coupling predicted by the physical theory.

(iii) The parameter $R$ from the physical theory coincides with $-2 \pi i t$, where $t$ is again the canonical parameter. Thus, the $q$-expansion of $\kappa_{t t t}$ in equation (8) will coincide with the asymptotic expansion in $R$ given by equation (9), evaluated on the generator $H^{\otimes 3}$ of $\operatorname{Sym}^{3} H^{-1,1}(\mathscr{M})$.

(iv) There is an explicit formula for the coefficients $\sigma_{k}$, as described below.

To explain the formula for $\sigma_{k}$, let $n_{k}$ denote the number of rational curves of degree $k$ on the generic quintic threefold. Candelas et al. propose the formula

$$
\kappa_{t t t}=5+\sum_{k=1}^{\infty} \frac{n_{k} k^{3} e^{2 \pi i k t}}{1-e^{2 \pi i k t}}=5+n_{1} e^{2 \pi i t}+\left(2^{3} n_{2}+n_{1}\right) e^{4 \pi i t}+\cdots
$$

which implicitly incorporates their expressions for the higher coefficients. (The first two expressions are $\left.\sigma_{1}\left(H^{\otimes 3}\right)=n_{1}, \sigma_{2}\left(H^{\otimes 3}\right)=2^{3} n_{2}+n_{1}.\right)$

In the large radius limit $\operatorname{Im} t \rightarrow \infty$, the right-hand side of equation (12) approaches 5 . This agrees with the mirror symmetry conjecture, ${ }^{5}$ since the topological intersection form on $\mathscr{M}$ is determined by its value on the standard generator $H$, viz., $H^{3}=5$.

Moreover, by comparing equations (11) and (12), we can predict values for the numbers $n_{k}$. The first two predictions are $n_{1}=2875$, which was classically known to be the number of lines on a quintic threefold, and $n_{2}=609250$, which coincides with the number of conics on a quintic threefold computed by Katz [22]. And the third prediction $n_{3}=317206375$ has just been verified by Ellingsrud and Strømme [16]!

How was formula (12) arrived at? I am told that the field theory computation necessary to derive this formula can be done in principle, but seems to be too hard to carry out in practice at present. So Candelas et al. give a rough derivation of this formula based on some assumptions. Why do they believe the resulting formula to be correct? I quote from [6]:

\footnotetext{
${ }^{5}$ This should not be taken as strong evidence in favor of the conjecture, since the definitions have been carefully designed to ensure that this limit would be correct.
} 
These numbers provide compelling evidence that our assumption about the form of the prefactor is in fact correct. The evidence is not so much that we obtain in this way the correct values for $n_{1}$ and $n_{2}$, but rather that the coefficients in eq. (11) have remarkable divisibility properties. For example asserting that the second coefficient $4,876,875$ is of the form $2^{3} n_{2}+n_{1}$ requires that the result of subtracting $n_{1}$ from the coefficient yields an integer that is divisible by $2^{3}$. Similarly, the result of subtracting $n_{1}$ from the third coefficient must yield an integer divisible by $3^{3}$. These conditions become increasingly intricate for large $k$. It is therefore remarkable that the $n_{k}$ calculated in this way turn out to be integers.

I would add that it is equally remarkable that the coefficients in eq. (11) themselves turn out to be integers: I know of no proof that this is the case.

These arguments have a rather numerological flavor. I am reminded of the numerological observations made by Thompson [38] and Conway and Norton [11] about the $j$-function and the monster group. At the time those papers were written, no connection between these two mathematical objects was known. The $q$-expansion of the $j$-function was known to have integer coefficients, and it was observed that these integers were integral linear combinations of the degrees of irreducible representations of the monster group. This prompted much speculation about possible deep connections between the two, but at the outset all such speculation had to be characterized as "moonshine" (Conway and Norton's term).

The formal similarities to the present work should be clear: a $q$-expansion of some kind is found to have integer coefficients, and these integers then appear to be integral linear combinations of another set of integers, which occur elsewhere in mathematics in a rather unexpected location. Perhaps it is too much to hope that the eventual explanation will be as pretty in this case.

\section{APPENDIX A: Proofs OF THE MONODROMY LEMMAS}

Let

$$
W_{0} \subset W_{1} \subset \cdots \subset W_{2 n}=H^{n}\left(X_{P^{\prime}}, \mathbb{Q}\right)
$$

be the monodromy weight filtration at $P$, and let

$$
F^{0} \supset F^{1} \supset \cdots \supset F^{n-1} \supset F^{n} \supset(0) \text {. }
$$

be the limiting Hodge filtration at $P$. (We refer the reader to [21] or [35] for the definitions.) By a theorem of Schmid [35], these induce a mixed Hodge structure on the cohomology. Note that since $N^{n+1}=0$, we have $W_{0}=\operatorname{Im} N^{n}$.

Moreover, if $\langle\mid\rangle$ denotes the polarization on the cohomology, we have

$$
\langle N x \mid y\rangle=-\langle x \mid N y\rangle .
$$


Recall also that the polarization is symmetric or skew-symmetric, depending on the dimension $n$ :

$$
\langle x \mid y\rangle=(-1)^{n}\langle y \mid x\rangle .
$$

Proof of Lemma 1. Since $W$. is the monodromy weight filtration, $N^{n}$ induces an isomorphism

$$
N^{n}: W_{2 n} / W_{2 n-1} \rightarrow W_{0}
$$

These spaces cannot be zero, since $\left(T_{P}-I\right)^{n} \neq 0$. On the other hand, since $F^{n+1}=(0)$, the Hodge structure on $W_{2 n} / W_{2 n-1}$ must be purely of type $(n, n)$. It follows that $F^{n} /\left(F^{n} \cap W_{2 n-1}\right)=W_{2 n} / W_{2 n-1}$. But since $F^{n}$ is one-dimensional, this can only happen if $F^{n} \subset W_{2 n}-W_{2 n-1}$, and $W_{2 n} / W_{2 n-1}$ has dimension one. By the isomorphism (13), $W_{0}=\operatorname{Im} N^{n}$ has dimension one as well.

Next, note that $W_{2 n-1} / W_{2 n-2}$ has a Hodge structure with two types, $(n$, $n-1)$ and $(n-1, n)$, each of which must determine a space of half the total dimension. But since $F^{n} \cap W_{2 n-1}=(0)$, nothing nonzero can have type $(n, n-1)$. It follows that $W_{2 n-1} / W_{2 n-2}=(0)$, and that $W_{1} / W_{0}=(0)$ as well (using the isomorphism induced by $N^{n-1}$ ).

Thus, the image of $N^{n-1}$ comes entirely from the map

$$
N^{n-1}: W_{2 n} \rightarrow W_{2}
$$

That this image is two-dimensional is easily seen: $W_{0}$ is one-dimensional, and there is an isomorphism

$$
N^{n-1}: W_{2 n} / W_{2 n-1} \rightarrow\left(\operatorname{Im} N^{n-1}\right) / W_{0},
$$

which shows that $\left(\operatorname{Im} N^{n-1}\right) / W_{0}$ is also one-dimensional. Q.E.D.

In order to prove Lemma 2, we must first prove

Lemma 3 (Essentially due to Friedman and Scattone [17]).

Good integral bases exist, and form bases of the two-dimensional $\mathbb{Q}$-vector space $\operatorname{Im} N^{n-1}$. If $g_{0}, g_{1}=\frac{1}{\lambda} N^{n-1} g$ is a good integral basis, then

$$
\frac{1}{\lambda} N^{n-1} x=-\left\langle g_{1} \mid x\right\rangle g_{0}+\left\langle g_{0} \mid x\right\rangle g_{1}
$$

for all $x \in H^{n}\left(X_{P^{\prime}}, \mathbb{Q}\right)$.

Proof. Choose either generator of $\operatorname{Im} N^{n} \cap H^{n}\left(X_{P^{\prime}}, \mathbb{Z}\right)$ as $g_{0}$. We claim that $\left(g_{0}\right)^{\perp}=W_{2 n-2}$. Let $h \in W_{2 n}-W_{2 n-2}$, so that $N^{n} h=a g_{0}$ with $a \neq 0$. Then for any $x$ we have

$$
\left\langle N^{n} x \mid h\right\rangle=(-1)^{n}\left\langle x \mid N^{n} h\right\rangle=(-1)^{n} a\left\langle x \mid g_{0}\right\rangle .
$$

Thus, $W_{2 n-2}=\operatorname{ker} N^{n} \subset\left(g_{0}\right)^{\perp}$. Since both $W_{2 n-2}$ and $\left(g_{0}\right)^{\perp}$ are codimension one subspaces of $W_{2 n}$, they must be equal. 
By Poincaré duality, the polarization on $H^{n}\left(X_{P^{\prime}}, \mathbb{Z}\right)$ is a unimodular pairing. Thus, there exists an element $g \in H^{n}\left(X_{P^{\prime}}, \mathbb{Z}\right)$ such that $\left\langle g_{0} \mid g\right\rangle=1$. Since $g \notin$ $\left(g_{0}\right)^{\perp}$, neither $N^{n-1} g$ nor $N^{n} g$ is zero. There is thus a unique positive rational number $\lambda$ such that $g_{1}=\frac{1}{\lambda} N^{n-1} g$ is an indivisible element of $H^{n}\left(X_{P^{\prime}}, \mathbb{Z}\right)$. It is clear that $g_{0}, g_{1}$ forms a basis for the $\mathbb{Q}$-vector space $\operatorname{Im} N^{n-1}$.

We next claim that $\left\langle g_{1} \mid g\right\rangle=\frac{1}{\lambda}\left\langle N^{n-1} g \mid g\right\rangle=0$. For, on the one hand, moving the $N$ 's to the right side one at a time we have

$$
\left\langle N^{n-1} g \mid g\right\rangle=(-1)^{n-1}\left\langle g \mid N^{n-1} g\right\rangle
$$

while, on the other hand, the symmetry of the polarization says that

$$
\left\langle N^{n-1} g \mid g\right\rangle=(-1)^{n}\left\langle g \mid N^{n-1} g\right\rangle \text {. }
$$

It follows that $\left\langle N^{n-1} g \mid g\right\rangle=0$.

To prove equation (14), we first compute in general

$$
\left\langle N^{n-1} x \mid g\right\rangle=(-1)^{n-1}\left\langle x \mid N^{n-1} g\right\rangle=(-1)^{n-1}\left\langle x \mid \lambda g_{1}\right\rangle=-\lambda\left\langle g_{1} \mid x\right\rangle \text {. }
$$

Now suppose that $x \in W_{2 n-2}$. Then $N^{n-1} x \in \operatorname{Im} N^{n}$, which implies that $N^{n-1} x=a g_{0}$ for some $a$. Thus, in this case

$$
\left\langle N^{n-1} x \mid g\right\rangle=\left\langle a g_{0} \mid g\right\rangle=a,
$$

which implies that $a=-\lambda\left\langle g_{1} \mid x\right\rangle$. Thus,

$$
\frac{1}{\lambda} N^{n-1} x=\frac{1}{\lambda} a g_{0}=\left\langle g_{1} \mid x\right\rangle g_{0}
$$

and since $\left\langle x \mid g_{0}\right\rangle=0$, the formula follows in this case.

To prove the formula in general, note that

$$
\left\langle g_{0} \mid x-\left\langle g_{0} \mid x\right\rangle g\right\rangle=0
$$

for any $x$, so that $x-\left\langle g_{0} \mid x\right\rangle g \in\left(g_{0}\right)^{\perp}=W_{2 n-2}$. Thus, applying the previous case we find

$$
\begin{aligned}
\frac{1}{\lambda} N^{n-1} x & =\frac{1}{\lambda} N^{n-1}\left(x-\left\langle g_{0} \mid x\right\rangle g\right)+\frac{1}{\lambda} N^{n-1}\left(\left\langle g_{0} \mid x\right\rangle g\right) \\
& =-\left\langle g_{1} \mid\left(x-\left\langle g_{0} \mid x\right\rangle g\right)\right\rangle g_{0}+\left\langle g_{0} \mid x\right\rangle \frac{1}{\lambda} N^{n-1} g \\
& =-\left(\left\langle g_{1} \mid x\right\rangle-\left\langle g_{0} \mid x\right\rangle\left\langle g_{1} \mid g\right\rangle\right) g_{0}+\left\langle g_{0} \mid x\right\rangle g_{1} \\
& =-\left\langle g_{1} \mid x\right\rangle g_{0}+\left\langle g_{0} \mid x\right\rangle g_{1}
\end{aligned}
$$

since $\left\langle g_{1} \mid g\right\rangle=0$. Q.E.D.

We can now prove Lemma 2.

Proof of Lemma 2. The only generators of $\operatorname{Im} N^{n} \cap H^{n}\left(X_{P^{\prime}}, \mathbb{Z}\right)$ are $\pm g_{0}$, so we must have $g_{0}^{\prime}=(-1)^{\ell} g_{0}$ for some $\ell \in \mathbb{Z}$. Write $g_{1}^{\prime}=\frac{1}{\lambda^{\prime}} N^{n-1} g^{\prime}$ for some 
$g^{\prime}$ with $\left\langle g_{0}^{\prime} \mid g^{\prime}\right\rangle=1$, and let $k=-\left\langle g_{1} \mid g^{\prime}\right\rangle \in \mathbb{Z}$. Then by Lemma 3,

$$
\frac{1}{\lambda} N^{n-1} g^{\prime}=-\left\langle g_{1} \mid g^{\prime}\right\rangle g_{0}+\left\langle g_{0} \mid g^{\prime}\right\rangle g_{1}=k g_{0}+(-1)^{\ell} g_{1} \text {. }
$$

Thus, $\frac{1}{\lambda} N^{n-1} g^{\prime} \in H^{n}\left(X_{P^{\prime}}, \mathbb{Z}\right)$. We claim that it must be an indivisible element there. For if $\frac{1}{\lambda \mu} N^{n-1} g^{\prime}$ is integral for some $\mu \in \mathbb{Z}$ with $\mu>1$, then reversing the roles of $g$ and $g^{\prime}$ in the argument above shows that $\frac{1}{\lambda \mu} N^{n-1} g$ is also integral, a contradiction.

$$
\text { Thus, } g_{1}^{\prime}=k g_{0}+(-1)^{\ell} g_{1} \text {. Q.E.D. }
$$

\section{APPENDIX B: RESOLUTIONS OF CERTAIN QUOTIENT SINGULARITIES}

In this appendix, we will verify that the singularities of the variety $\mathscr{Q}_{\psi} / G$ can be resolved in a natural way. The choices we make are sufficiently natural that the isomorphism between $\mathscr{Q}_{\alpha \psi} / G$ and $\mathscr{Q}_{\psi} / G$ automatically lifts to an isomorphism between the desingularizations.

We choose to follow the strategy outlined by Reid [30] for resolving canonical threefold singularities. In brief, we perform the following steps:

Step I: Blow up the "non-cDV points" of $\mathscr{Q}_{\psi} / G$. (These are exactly the 10 points $p_{i j} \in \mathscr{Q}_{\psi} / G$ which are the images of points in $\mathscr{Q}_{\psi}$ with stabilizer of order 25.)

Step IIA: Blow up the singular locus. (It has pure dimension one.)

Step IIB: Blow up the pure dimension one part of the singular locus. $(60$ isolated singular points (lying over the $p_{i j}$ ) were created by step IIA, and these are not to be blown up yet.)

Step III: Obtain a projective small resolution of the remaining 60 singular points by blowing up the union of the proper transforms of the exceptional divisors from step I.

Step III involved an additional choice, since Reid's strategy does not specify how one should obtain small resolutions.

When stated in this form, it is clear that the process we have described is sufficiently natural that it is preserved under any isomorphism. It yields a projective (hence Kähler) variety $\mathscr{W}_{\psi}$ with trivial canonical bundle.

In the remainder of this appendix, we will show that the process above has the properties mentioned during its description, and that it gives a resolution of singularities of $\mathscr{Q}_{\psi} / G$.

We first observe the effect of the process on the curve $C_{i j k}$, away from the points $p_{i j}, p_{j k}, p_{i k}$. Steps I and III are concentrated at those special points (and their inverse images) and so these steps do not affect $C_{i j k}$. Steps IIA and IIB simply blow up $C_{i j k}$ and then the residual singular curve in the exceptional divisor. But two blowups are precisely what is required to resolve a rational double point of type $A_{4}$, as is easily verified from its equation $x y+z^{5}=0$.

To verify that the process has the correct properties at the points $p_{i j}$, we use the language of toroidal embeddings (see [23] or [29]). It suffices to consider the 
point $p_{45}$. Since $\left(x_{1}, x_{2}, x_{3}\right)$ serve as coordinates in a neighborhood of any of the points in $\eta^{-1}\left(p_{45}\right)$, the singularity $p_{45} \in \mathscr{Q}_{\psi} / G$ is isomorphic to a neighborhood of the origin in $\mathbb{C}^{3} / G_{45}$, where $G_{45} \cong\left\{\left(\alpha_{1}, \alpha_{2}, \alpha_{3}\right) \in\left(\boldsymbol{\mu}_{5}\right)^{3} \mid \prod \alpha_{k}=1\right\}$ acts diagonally on $\mathbb{C}^{3}$.

Let $M$ be the lattice of $G_{45}$-invariant rational monomials in $\mathbb{C}\left(x_{1}, x_{2}, x_{3}\right)$. We embed $M$ in $\mathbb{R}^{3}$ by ${ }^{6}$

$$
M=\left\{\left(m_{1}, m_{2}, m_{3}\right) \in \mathbb{R}^{3} \mid x_{1}^{5 m_{1}} x_{2}^{5 m_{2}} x_{3}^{5 m_{3}} \in \mathbb{C}\left(x_{1}, x_{2}, x_{3}\right)^{G_{45}}\right\} .
$$

It is easy to see that $\left\{(1,0,0),(0,1,0),\left(\frac{1}{5}, \frac{1}{5}, \frac{1}{5}\right)\right\}$ is a basis of the lattice $M \subset \mathbb{R}^{3}$. Let

$$
\begin{aligned}
N & =\left\{\vec{n} \in \mathbb{R}^{3} \mid \vec{m} \cdot \vec{n} \in \mathbb{Z} \text { for all } \vec{m} \in M\right\} \\
& =\left\{\left(n_{1}, n_{2}, n_{3}\right) \in \mathbb{Z}^{3} \mid n_{1}+n_{2}+n_{3} \equiv 0 \bmod 5\right\}
\end{aligned}
$$

be the dual lattice, and let $\sigma \subset N_{\mathbb{R}}$ be the convex cone generated by $(5,0,0)$, $(0,5,0)$, and $(0,0,5)$. According to the theory of toroidal embeddings,

$$
\mathbb{C}^{3} / G_{45}=\operatorname{Spec} \mathbb{C}\left[x_{1}, x_{2}, x_{3}\right]^{G_{45}}=U_{\sigma},
$$

where $U_{\sigma}$ is the toric variety associated to $\sigma$.

Each blowup of $U_{\sigma}$ corresponds to a decomposition of $\sigma$ into a fan. The effects of the blowups in our process is illustrated in Figure 1, which depicts the intersection of the fan with $\left\{\left(n_{1}, n_{2}, n_{3}\right) \in N_{\mathbb{R}} \mid \sum n_{k}=5\right\}$ after each step. The exceptional divisors $D_{\vec{n}}$ of each blowup are indicated by solid dots, labeled by the corresponding elements $\vec{n} \in N$. (The fact that the stated blowups produce the illustrated decomposition is a straightforward calculation with the toroidal embeddings.)

We can now see in detail what happens in our process. In step I, we blow up $p_{45}$, and produce three exceptional divisors $D_{(3,1,1)}, D_{(1,3,1)}$, and $D_{(1,1,3)}$. The remaining singular locus at this stage consists of the original three curves of $A_{4}$-singularities together with three new curves of $A_{1}$-singularities: the intersections of pairs of exceptional divisors. In step IIA, we blow up the union of these six curves, and produce nine new exceptional divisors: one corresponding to each curve of $A_{1}$-singularities (such as $D_{(2,1,2)}$ ), and two corresponding to each curve of $A_{4}$-singularities (such as $D_{(4,0,1)}$ and $D_{(1,0,4)}$ ). The remaining singularities consist of six isolated points (corresponding to the quadrilaterals in the figure) and three curves: the intersections of the corresponding pairs of exceptional divisors from the original $A_{4}$-singularities.

In step IIB, we blow up these three curves, producing six new exceptional divisors, two for each curve (such as $D_{(3,0,2)}$ and $\left.D_{(2,0,3)}\right)$. This leaves the six isolated singular points; but blowing up the proper transforms of $D_{(3,1,1)}$,

\footnotetext{
${ }^{6}$ This nonstandard embedding is chosen in order to make the coordinates of the dual lattice be integers.
} 


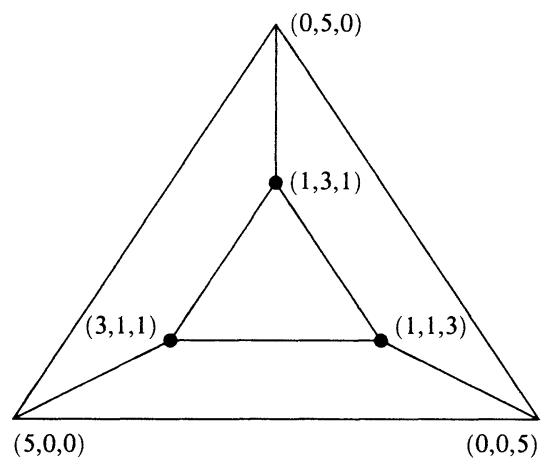

Step I

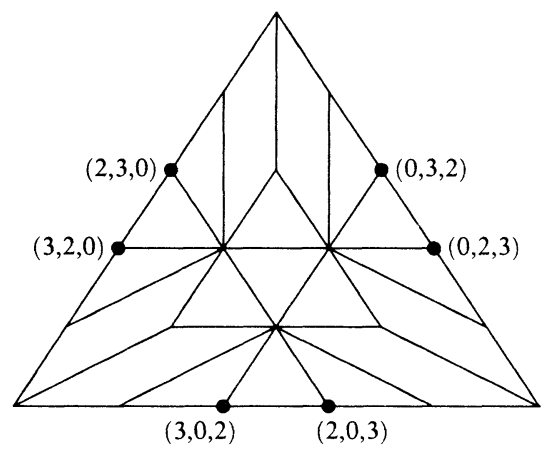

Step IIB

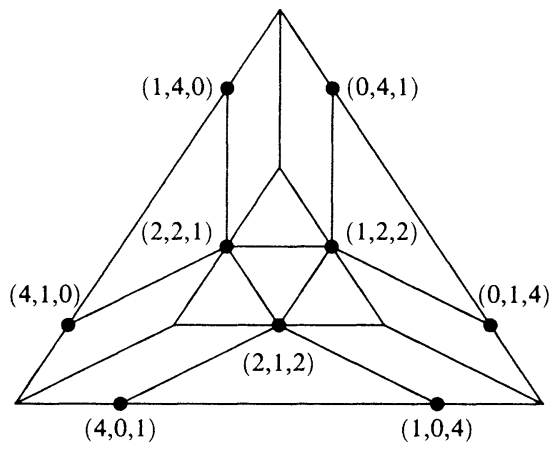

Step IIA

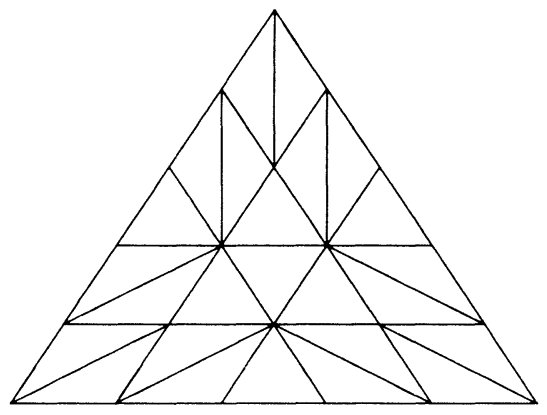

Step III

FigURE 1. The steps in the toroidal resolution.

$D_{(1,3,1)}$, and $D_{(1,1,3)}$ (which are now disjoint) in step III resolves those final singular points.

\section{APPENDIX C: THE MONODROMY OF THE QUINTIC-MIRRORS}

In this appendix we will explain how to use the calculation of Candelas et al. [6] to verify the monodromy statements about the family of quintic-mirrors which we made in $\S 5$.

Candelas et al. begin by choosing an explicit basis $\left\{A^{1}, A^{2}, B_{1}, B_{2}\right\}$ for the homology $H_{3}\left(\mathscr{W}_{\psi}, \mathbb{Z}\right)$ of a quintic-mirror, valid in some simply-connected region in $\left\{\psi \mid \psi^{5} \neq 0,1\right\}$ which includes the wedge $\{\psi \mid 0<\arg \psi<2 \pi / 5\}$. This basis is symplectic, i.e., $A^{a} \cap B_{b}=\delta^{a}{ }_{b}$ and $A^{a} \cap A^{b}=B_{a} \cap B_{b}=0$. The corresponding dual basis of $H^{3}\left(\mathscr{W}_{\psi}, \mathbb{Z}\right)$ is denoted by $\left\{\alpha_{1}, \alpha_{2}, \beta^{1}, \beta^{2}\right\}$. Fixing a particular holomorphic 3-form $\Omega$ (which depends on $\psi$ ), we then get period functions

$$
z^{a}=\int_{A^{a}} \Omega, \quad \mathscr{G}_{b}=\int_{B_{b}} \Omega .
$$


These fit into a period vector

$$
\Pi=\left(\begin{array}{l}
\mathscr{G}_{1} \\
\mathscr{G}_{2} \\
z^{1} \\
z^{2}
\end{array}\right) .
$$

By doing some integrals, calculating the differential equation satisfied by a period function, and manipulating certain hypergeometric functions, the authors of [6] are able to obtain explicit formulas for the four period functions. This allows them to calculate the monodromy of the periods around various paths.

Notice that we are working in the $\psi$-plane at present. The family $\left\{\mathscr{W}_{\psi}\right\}$ has singular fibers at $\psi=0$ and at $\psi=\alpha$ for all fifth roots of unity $\alpha$; there is also a singular fiber over $\psi=\infty$. Candelas et al. calculate the monodromy on the periods induced by transport around $\psi=1$, which they represent in matrix form by $\Pi \rightarrow T \Pi$. They also compute, for $|\psi|<1$, the effect on the periods of the isomorphism $\mathscr{W}_{\alpha \psi} \cong \mathscr{W}_{\psi}$ given in equation (10), representing this by $\Pi(\alpha \psi)=A \Pi(\psi)$.

We need to know the monodromy around $\infty$ in the $\lambda$-plane, where $\lambda=\psi^{5}$. A moment's thought will convince the reader that this is represented by

$$
\Pi \rightarrow\left(T^{-1} A^{-1}\right) \Pi
$$

and that $(A T)^{-5}$ describes the monodromy around $\infty$ in the $\psi$-plane (as asserted in [6]). Let $T_{P}=T^{-1} A^{-1}$.

The explicit calculations from [6] for the matrices $A$ and $T$ are:

$$
A=\left(\begin{array}{rrrr}
-9 & -3 & 5 & 3 \\
0 & 1 & 0 & -1 \\
-20 & -5 & 11 & 5 \\
-15 & 5 & 8 & -4
\end{array}\right), \quad T=\left(\begin{array}{llll}
1 & 0 & 0 & 0 \\
0 & 1 & 0 & 1 \\
0 & 0 & 1 & 0 \\
0 & 0 & 0 & 1
\end{array}\right)
$$

from which it easily follows that

$$
\left(\log \left(T_{P}\right)\right)^{2}=\left(\begin{array}{rrrr}
0 & 5 & 0 & 0 \\
0 & 0 & 0 & 0 \\
0 & 10 & 0 & 0 \\
-10 & 0 & 5 & 0
\end{array}\right), \quad\left(\log \left(T_{P}\right)\right)^{3}=\left(\begin{array}{rrrr}
0 & 0 & 0 & 0 \\
0 & 0 & 0 & 0 \\
0 & 0 & 0 & 0 \\
0 & -5 & 0 & 0
\end{array}\right)
$$

In particular, the index of nilpotency of $\log \left(T_{P}\right)$ is maximal.

(We note in passing that at $\lambda=1$ the monodromy is represented by $T$, and since $(T-I)^{2}=0$, the index is not maximal there. In addition, at $\lambda=0$ the monodromy is represented by $A$. This monodromy matrix is only quasiunipotent, with $A^{5}=I$ unipotent; the index of $A^{5}$ is not maximal either. It follows that $\lambda=\infty$ is the only possible boundary point with maximally unipotent monodromy.) 
In order to construct a good integral basis $g_{0}, g_{1}$, we compute

$$
\begin{aligned}
& \left(\log \left(T_{P}\right)\right)^{2}\left(\int_{A^{2}} \Omega\right)=-10 \int_{B_{1}} \Omega+5 \int_{A^{1}} \Omega, \\
& \left(\log \left(T_{P}\right)\right)^{3}\left(\int_{A^{2}} \Omega\right)=-5 \int_{B_{2}} \Omega .
\end{aligned}
$$

Using the relations

$$
\operatorname{ad}_{\langle\mid\rangle}\left(\alpha_{a}\right)=\int_{B_{a}}, \quad \operatorname{ad}_{\langle\mid\rangle}\left(\beta^{b}\right)=-\int_{A^{b}},
$$

this implies

$$
\begin{aligned}
& \left(\log \left(T_{P}\right)\right)^{2}\left(\beta^{2}\right)=10 \alpha_{1}+5 \beta^{1} \\
& \left(\log \left(T_{P}\right)\right)^{3}\left(\beta^{2}\right)=5 \alpha_{2} .
\end{aligned}
$$

Thus, we may take $g_{0}=\alpha_{2}$. If we then choose $g=\beta^{2}$ so that $\left\langle g_{0} \mid g\right\rangle=$ $\left\langle\alpha_{2} \mid \beta^{2}\right\rangle=1$, we get $\lambda=5$ and $g_{1}=2 \alpha_{1}+\beta^{1}$. It follows that

$$
\left(\log \left(T_{P}\right)\right)\left(g_{1}\right)=\frac{1}{5}\left(\log \left(T_{P}\right)\right)^{3}\left(\beta^{2}\right)=\alpha_{2}=g_{0},
$$

which implies that $m=1$. Using the relations (15) again, it follows that $\gamma_{0}=B_{2}, \gamma_{1}=2 B_{1}-A^{1}$. Thus, $t=\left(\int_{2 B^{1}-A_{1}} \Omega\right) /\left(\int_{A^{2}} \Omega\right)$.

We need to verify that our parameter $t$ is the same one used by Candelas et al. Their parameter is defined in $[6,(5.9)]$ by $t=w^{1} / w^{2}$, with $w^{1}$ and $w^{2}$ determined by a pair of equations

$$
\amalg=N \Pi, \quad w^{2}=\mathscr{G}_{2},
$$

where $^{7}$

$$
\amalg=\left(\begin{array}{c}
\mathscr{F}_{1} \\
\mathscr{F}_{2} \\
w^{1} \\
w^{2}
\end{array}\right) \text { and } N=\left(\begin{array}{rrrr}
-1 & 0 & 0 & 0 \\
0 & 0 & 0 & 1 \\
2 & 0 & -1 & 0 \\
0 & 1 & 0 & 0
\end{array}\right)
$$

represent a vector $\amalg$ which is a sort of mirror analogue of the period vector $\Pi$, and a particular integral symplectic matrix $N$, respectively. (Sadly, the symbols $\Pi$ and $\amalg$ were inadvertently identified in [6, section 5.2]; the correct symbols can be deduced from the brief version of [6] in Physics Letters.) It follows that

$$
t=\frac{w^{1}}{w^{2}}=\frac{2 \mathscr{G}_{1}-z^{1}}{\mathscr{G}_{2}}=\frac{\int_{2 B^{1}-A_{1}} \Omega}{\int_{A^{2}} \Omega}
$$

as required.

\footnotetext{
[6].

${ }^{7}$ We have taken the liberty of correcting a typographical error in $N$ when transcribing it from
} 


\section{ACKNOWLEDGMENTS}

It is a pleasure to acknowledge helpful conversations and e-mail exchanges with Paul Aspinwall, Robert Bryant, Philip Candelas, Brian Greene, Yujiro Kawamata, Ronen Plesser, Les Saper, Chad Schoen, and especially Sheldon Katz as I was struggling to understand this material.

\section{REFERENCES}

1. P. S. Aspinwall and C. A. Lütken, Geometry of mirror manifolds, Nuclear Phys. B 353 (1991), 427-461.

2. _ Quantum algebraic geometry of superstring compactifications, Nuclear Phys. B 355 (1991), 482-510.

3. P. S. Aspinwall, C. A. Lütken, and G. G. Ross, Construction and couplings of mirror manifolds, Phys. Lett. B 241 (1990), 373-380.

4. F. A. Bogomolov, Hamiltonian Kähler manifolds, Dokl. Akad. Nauk SSSR 243 (1978), no. 5, 1101-1104.

5. P. Candelas, Yukawa couplings between $(2,1)$-forms, Nuclear Phys. B 298 (1988), 458-492.

6. P. Candelas, X. C. de la Ossa, P. S. Green, and L. Parkes, A pair of Calabi-Yau manifolds as an exactly soluble superconformal theory, Phys. Lett. B 258 (1991), 118-126; Nuclear Phys. B 359 (1991), 21-74.

7. P. Candelas, M. Lynker, and R. Schimmrigk, Calabi-Yau manifolds in weighted $\mathbb{P}_{4}$, Nuclear Phys. B 341 (1990), 383-402.

8. J. Carlson, M. Green, P. Griffiths, and J. Harris, Infinitesimal variations of Hodge structure. I, Compositio Math. 50 (1983), 109-205.

9. S. Cecotti, $N=2$ supergravity, type IIB superstrings, and algebraic geometry, Comm. Math. Phys. 131 (1990), 517-536.

10. $\quad N=2$ Landau-Ginzburg vs. Calabi-Yau $\sigma$-models: Non-perturbative aspects, Internat. J. Modern Phys. A 6 (1991), 1749-1813.

11. J. H. Conway and S. P. Norton, Monstrous moonshine, Bull. London Math. Soc. 11 (1979), 308-339.

12. R. Dijkgraaf, E. Verlinde, and H. Verlinde, On moduli spaces of conformal field theories with $c \geq 1$, Perspectives in String Theory (P. Di Vecchia and J. L. Petersen, eds.), World Scientific, Singapore, New Jersey, and Hong Kong, 1988, pp. 117-137.

13. M. Dine, N. Seiberg, X.-G. Wen, and E. Witten, Nonperturbative effects on the string world sheet. II, Nuclear Phys. B 289 (1987), 319-363.

14. J. Distler and B. Greene, Some exact results on the superpotential from Calabi-Yau compactifications, Nuclear Phys. B 309 (1988), 295-316.

15. L. J. Dixon, Some world-sheet properties of superstring compactifications, on orbifolds and otherwise, Superstrings, Unified Theories, and Cosmology 1987 (G. Furlan et al., eds.), World Scientific, Singapore, New Jersey, and Hong Kong, 1988, pp. 67-126.

16. G. Ellingsrud and S. A. Strømme, The number of twisted cubic curves on the general quintic threefold, University of Bergen Report no. 63-7-2-1992.

17. R. Friedman and F. Scattone, Type III degenerations of K3 surfaces, Invent. Math. 83 (1986), $1-39$.

18. D. Gepner, Exactly solvable string compactification on manifolds of $S U(N)$ holonomy, Phys. Lett. B 199 (1987), 380-388.

19. B. R. Greene and M. R. Plesser, Duality in Calabi-Yau moduli space, Nuclear Phys. B 338 (1990), 15-37.

20. B. R. Greene, C. Vafa, and N. P. Warner, Calabi-Yau manifolds and renormalization group flows, Nuclear Phys. B 324 (1989), 371-390.

21. P. Griffiths (ed.), Topics in transcendental algebraic geometry, Ann. of Math. Stud., vol. 106, Princeton University Press, Princeton, NJ, 1984. 
22. S. Katz, On the finiteness of rational curves on quintic threefolds, Compositio Math. 60 (1986), 151-162.

23. G. Kempf, F. Knudsen, D. Mumford, and B. Saint-Donat, Toroidal embeddings. I, Lecture Notes in Math., vol. 339, Springer-Verlag, Berlin, Heidelberg, and New York, 1973.

24. A. Landman, On the Picard-Lefschetz transformations, Trans. Amer. Math. Soc. 181 (1973), 89-126.

25. W. Lerche, C. Vafa, and N. P. Warner, Chiral rings in $N=2$ superconformal theories, Nuclear Phys. B 324 (1989), 427-474.

26. D. G. Markushevich, Resolution of singularities (toric method), appendix to: D. G. Markushevich, M. A. Olshanetsky, and A. M. Perelomov, Description of a class of superstring compactifications related to semi-simple Lie algebras, Comm. Math. Phys. 111 (1987), 247274.

27. E. J. Martinec, Algebraic geometry and effective Lagrangians, Phys. Lett. B 217 (1989), 431-437.

28. Criticality, catastrophes, and compactifications, Physics and Mathematics of Strings (L. Brink, D. Friedan, and A. M. Polyakov, eds.), World Scientific, Singapore, New Jersey, London, and Hong Kong, 1990, pp. 389-433.

29. T. Oda, Convex bodies and algebraic geometry, Ergeb. Math. Grenzgeb. (3), Bd. 15, SpringerVerlag, Berlin, Heidelberg, and New York, 1988.

30. M. Reid, Minimal models of canonical 3-folds, Algebraic Varieties and Analytic Varieties (S. Iitaka, ed.), Kinokuniya, Tokyo, and North-Holland, Amsterdam, 1983, pp. 131-180.

31. Ann. 278 (1987), 329-334.

32. S.-S. Roan, On the generalization of Kummer surfaces, J. Differential Geom. 30 (1989), 523-537.

33. , On Calabi-Yau orbifolds in weighted projective spaces, Internat. J. Math. 1 (1990), 211-232.

34. _ The mirror of Calabi-Yau orbifold, Max-Planck-Institut preprint MPI/91-1, Internat. J. Math. 2 (1991), 439-455.

35. W. Schmid, Variation of Hodge structure: the singularities of the period mapping, Invent. Math. 22 (1973), 211-319.

36. C. Schoen, On the geometry of a special determinental hypersurface associated to the Mumford-Horrocks vector bundle, J. Reine Angew. Math. 364 (1986), 85-111.

37. A. Strominger and E. Witten, New manifolds for superstring compactification, Comm. Math. Phys. 101 (1985), 341-361.

38. J. G. Thompson, Some numerology between the Fischer-Griess monster and the elliptic modular function, Bull. London Math. Soc. 11 (1979), 352-353.

39. G. Tian, Smoothness of the universal deformation space of compact Calabi-Yau manifolds and its Peterson-Weil metric, Mathematical Aspects of String Theory (S. T. Yau, ed.), World Scientific, Singapore, 1987, pp. 629-646.

40. A. N. Todorov, The Weil-Petersson geometry of the moduli space of $S U(n \geq 3)$ (Calabi-Yau) manifolds. I, Comm. Math. Phys. 126 (1989), 325-246.

41. S. T. Yau, On Calabi's conjecture and some new results in algebraic geometry, Proc. Nat. Acad. Sci. U.S.A. 74 (1977), 1798-1799.-60pt

ABSTRACT. We give a mathematical account of a recent string theory calculation which predicts the number of rational curves on the generic quintic threefold. Our account involves the interpretation of Yukawa couplings in terms of variations of Hodge structure, a new $q$-expansion principle for functions on the moduli space of Calabi-Yau manifolds, and the "mirror symmetry" phenomenon recently observed by string theorists.

Department of Mathematics, Duke University, Durham, North Carolina 27706

E-mail address: drm@math.duke.edu 